\title{
Adenocarcinoma of the Appendix in Young Men with Acute Appendicitis
}

\author{
K. Alexiou, Argyrios Ioannidis*, I. Drikos, I. Karanikas, \\ A. Fotopoulos, M. Chorti, N. Economou \\ Department of surgery, Sismanoglion General Hospital, Athens, Greece \\ Email: argioannid@med.uoa.gr
}

Received 8 April 2014; revised 2 May 2014; accepted 10 May 2014

Copyright (C) 2014 by authors and Scientific Research Publishing Inc. This work is licensed under the Creative Commons Attribution International License (CC BY). http://creativecommons.org/licenses/by/4.0/

(c) (i) Open Access

\begin{abstract}
Intoduction: The adenocarcinomas of the appendix are rare tumors of the gastrointestinal truct that often reveal no clear clinical symptoms and are not diagnosed in time. In this study, we analyze a rare case of an incidentally discovered adenocarcinoma of the appendix after appendicectomy. Case presentation: A Greek male patient of 37 years old patient presented to our hospital with signs of acute appendicitis and peritonitis. He underwent appendicectomy as therapeutic treatment. Post operational histological examination revealed in situ carcinoma of the appendix without high hematological tumor markers or visible metastasis in CT scan analysis. We decided to proceed to right hemicolectomy. The patient was discharged without complications and without displaying other symptoms in a monitoring interval of two months after surgery. Conclusion: Adenocarcinomas of the appendix are generally difficult to detect in the early stage leading to significant morbidity and mortality rates. Therefore, it is necessary to execute a very careful histopathological examination and perform a detailed intraperitoneal cleaning during operation, so that any indications of appendix adenocarcinomas in cases of appendectomy won't be missed or misjudged.
\end{abstract}

\section{Keywords}

Acute Appendicitis, Appendix Adenocarcinoma, Right Hemicolectomy

\section{Introduction}

The tumors of the appendix are rare carcinomas of the gastrointestinal tracts, most of which are crustaceans, while a small percentage are mucous cystadenocarcinomas, adenocarcinomas, lymphosarcomas, paragangliomas

"Corresponding author. 
and granular cell tumors. The main adenocarcinoma symptoms include acute abdominal pain in the right iliac fossa, signs of peritoneal inflammation, fever, leukocytosis, resulting in the clinical diagnosis of acute appendicitis to lay the foundations of surgical removal of the appendix. In our case, the appendix carcinoma therapy included the right hemicolectomy, with resection of the related lymph nodes, the same as for the common forms of colon cancer.

\section{Case Presentation}

In this clinical case we analyze a Greek male patient of 37 years old with no previous abdominal surgeries, no alcohol intake, non-smoker and no allergies. He just reports hyperuricemia under pharmacological treatment. He presented to the emergency department with signs of pain in the right lower quadrant (right iliac fossa), tachycardia, positive McBurney point, fever and no symptoms of vomiting, diarrhea or bloody stools.

The laboratory blood tests revealed mild leukocytosis (white blood cell count of $11.800 / \mathrm{mm}^{3}$ ) with polymorphonuclear type, while the general urine test did not reveal inflammation of the urinary tract. Also blood tests revealed elevated liver enzymes alanine aminotransferase, $85 \mathrm{U} / \mathrm{L}$ (normal 0 - 50); aspartate aminotransferase, $51 \mathrm{U} / \mathrm{L}$ (normal 0 - 40); c-glutamyl transpeptidase, $128 \mathrm{U} / \mathrm{L}$ (normal 0 - 40); alkaline phosphatase, $136 \mathrm{IU} / \mathrm{L}$ (normal 30 - 140); and CRP $172.9 \mathrm{mg} / \mathrm{dl}$ (normal 0 - 10).

We clinically diagnosed acute appendicitis and we performed surgery for resection of the appendix. During the surgical procedure, we found the appendix broken into 3 (three) segments and showed signs of serious inflammation. We also recognized peritoneal abscess and therefore before the end of the surgery we cleaned the abdomen carefully and placed 2 (two) drainage tubes, one in the anatomical position of the appendix and the second one at the pouch of Douglas. The patient remained at non per os diet for 2 days after surgery. He underwent IV fluids and antibiotics (Ciprofloxacin $400 \mathrm{mg}$ S: $1 \times 3$, Metronidazole $500 \mathrm{mg}$ S: $1 \times 3$ ). His temperature returned to normal the second post-operational day and he also started walking the second post-operational day. He exited the hospital, with no fever, no pain or any other symptoms at the sixth $\left(6^{\text {th }}\right)$ post-operational day after appendicectomy and peritoneal cleaning.

Upon histological examination of the segments of the appendix, the coatings were brittle while the examined sections showed mucosal type papillary adenomas with high-grade epithelial dysplasia and carcinomatous transformation, with focal infiltration of mucosal muscle layer (Figure 1). Due to high dysplasia and carcinomatous transformation, the patient was contacted and re-entered the hospital exactly 3 weeks after his exit. He was operated for a second time, this time for a right hemicolectomy. Right ureter catheter was placed during the procedure to secure the right ureter. Patient stayed in the hospital for a total of 5 days. He exited the hospital in perfect condition with no symptoms at all.

Until today (6 months after second operation) he remains asymptomatic. His Computed Scan Tomography shows no signs of metastasis.

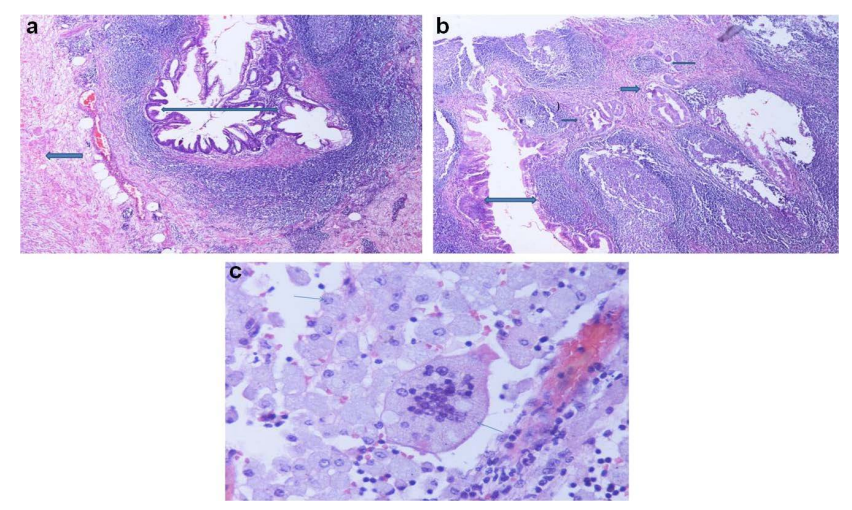

Figure 1. (a) Well differentiated mucinous appendiceal adenocarcinoma arising from vilous adenoma (double narrow) and appendix muscular wall (simple narrow), H-EX100; (b) low grade appendix adenorcarcinoma (double narrow) and infiltrated muscularis mucosae (simple arrows), H-EX100 and (c) Mucin phagocytosis from histiocytes or multinucleated giant cells in the right hemicolectomy specimen (arrows), H-EX 400. 


\section{Discussion}

The tumors of the appendix are rare tumors of the gastrointestinal truct and in most cases are crustaceans, while few of them are mucous cystadenocarcinomas, adenocarcinomas, lymphosarcomas, paragangliomas and granular cell tumors. The main symptoms include acute abdominal pain in the right iliac fossa, signs of peritoneal inflammation, fever, leukocytosis [1].

Adenocarcinomas refer to less than $10 \%$ of the GI tumors [2], while benign tumors are the most frequent adenomas and benign neoplastic mucocele [3]. The cases of tumors of the appendix are diagnosed after appendicectomy by histological analysis and rarely give any symptoms and signs either by clinical examination or by hematological indices [4] [5].

The proper selected operation after the diagnosis of carcinoma of the appendix is hemicolectomy appearing 5 year survival rate up to 67\% [6] [7]. Park et al. calculate the total 5-year survival rate after surgical excision of adenocarcinoma and lymph nodes removal to $20.5 \%$ [8].

Furthermore, there are certain neoplasmatic cases which histological examination is indicative, while it is becoming indispensable not only for cases of appendicectomy in patients presenting symptoms of acute appendicitis, but also in any other surgical procedure [9].

In cases of appendicectomy, the surgical removal must be followed by careful observation of the base of the appendix along with the histological examination of the appendix in order to avoid the presence of any other lesions of the colon which have multifocal configuration [10].

The hemicolectomy therefore is considered to be the proper treatment in cases of carcinoma lesions extending beyond the mucosa. While some scientists suggest that the cases of in situ carcinomas should be treated by local colectomy only at the area of the appendix and around tissues. Varisco et al. in a meta-analysis of 100 patients with localized cases of appendiceal carcinoid and low differentiation conclude that only in these cases of tumors can be assessed by this surgical practice [11].

Additionally, proposed application of postoperative chemotherapy could be appropriate for cases of peritoneal implantation of tumor cells in combine with peritoneum therapeutic approaches such as surgical treatment of the peritoneum [12] [13]. Carcinomas of the appendix may have a variety of clinical symptoms that may be associated with the tumor size and cellular atypia such as bladder symptoms [14] or in some cases unilateral ureteral obstruction [15] and bilateral obstruction of both ureters due to the size of the tumor [16].

\section{Conclusion}

Adenocarcinoma of the appendix is a rare tumor that cannot be diagnosed easily and carries out a significant rate of mortality and morbidity even in young patients. The proper therapeutic approach tends to be right hemicolectomy. Despite surgery and adjuvant therapy, the prognosis of tumors of the appendix is poor. Careful histopathological examination and cleaning of the abdomen are necessary after appendicectomy.

\section{Acknowledgements}

We would like to thank the consultants of the Department of Surgery, Sismanoglion general hospital Athens, for kindly allowing their patients to be included in the series.

\section{Consent}

Written informed consent was obtained from the patient for publication of this case report and any accompanying images. A copy of the written consent is available for review by the Editor-in-Chief of this journal.

\section{Authors' Contributions}

Konstantinos Alexiou, Argyrios Ioannidis and Economou Nikolaos carried out and participated in the surgical excision and the manuscript demonstration. All authors participated in the design of the study and helped to draft the manuscript. All authors read and approved the final manuscript.

\section{Declaration of Competing Interests}

The authors declare that they have no competing interests. 


\section{References}

[1] McCusker, M., Coté, T., Clegg, L. and Sobin, L. (2002) Primary Malignant Neoplasms of the Appendix: A Population Based Study from the Surveillance, Epidemiology and Endresults Program, 1973-1998. Cancer, 94, 3307-3312. http://dx.doi.org/10.1002/cncr.10589

[2] Connor, S., Hanna, G. and Frizelle, F. (1998) Retrospective Clinicopathologic Analysis of Appendiceal Tumors from 7970 Appendectomies. Diseases of the Colon and Rectum, 41, 75-80. http://dx.doi.org/10.1007/BF02236899

[3] Ruoff, C., Hanna, L., Zhi, W., Shahzad, G., Gotlieb, V. and Saif, M. (2011) Cancers of the Appendix: Review of the Literatures. International Scholarly Research Network (ISRN) Oncology, 2011, Article ID: 728579.

[4] O’Donnell, M., Badger, S., Beattie, G., Carson, J. and Garstin, W. (2007) Malignant Neoplasms of the Appendix. International Journal of Colorectal Disease, 22, 1239-1248. http://dx.doi.org/10.1007/s00384-007-0304-0

[5] Nilecki, S., Wolff, B., Schlinkert, R. and Sarr, M. (1994) The Natural History of Surgically Treated Primary Adenocarcinoma of the Appendix. Annals of Surgery, 219, 51-57. http://dx.doi.org/10.1097/00000658-199401000-00009

[6] Murphy, E.M., Farquharson, S.M. and Moran, B.J. (2006) Management of an Unexpected Appendiceal Neoplasm. British Journal of Surgery, 93, 783-792. http://dx.doi.org/10.1002/bjs.5385

[7] Park, I., Yu, C., Kim, H. and Kim, J. (2004) Clinical Features and Prognostic Factors in Primary Adenocarcinoma of the Appendix. The Korean Journal of Gastroenterology, 43, 29-34.

[8] Turaga, K., Pappas, S. and Gamblin, C. (2013) Right Hemicolectomy for Mucinous Adenocarcinoma of the Appendix: Just Right or Too Much? Annals of Surgical Oncology, 20, 1063-1067. http://dx.doi.org/10.1245/s10434-012-2783-7

[9] Swank, H., Eshuis, E., Ubbink, D. and Bemelman, W. (2011) Is Routine Histopathological Examination of Appendectomy Specimens Useful? A Systematic Review of the Literature. Colorectal Disease, 13, 1214-1221. http://dx.doi.org/10.1245/s10434-012-2783-7

[10] Trivedi, A., Levine, E.A. and Mishra, G. (2009) Adenocarcinoma of the Appendix Is Rarely Detected by Colonoscopy. Journal of Gastrointestinal Surgery, 13, 668-675. http://dx.doi.org/10.1007/s11605-008-0774-6

[11] Varisco, B., McAlvin, B., Dias, J. and Franga, D. (2004) Adenocarcinoid of the Appendix: Is Right Hemicolectomy Necessary? A Meta-Analysis of Retrospective Chart Reviews. The American Surgeon, 70, 593-599.

[12] Smejkal, P., Pazdro, A., Smejkal, M., Pafko, P. and Frantlova, M. (2005) The Cystadenocarcinoma of the Appendix. Rozhledy $v$ Chirurgii, 84, 33-36.

[13] Vaira, M., Scuderi, S., Costamagna, B.R., Aghemo, B., Mioli, P.R. and De Simone, M. (2001) Cytoreductive Surgery and Intraperitoneal Hyperthermic Antiblastic Therapy (HAPP) in Peritoneal Carcinomatosis. Minerva Chirurgica, 57, 597-605.

[14] Mori, N., Noma, M., Hara, T., Yamaguchi, S., Shibata, K., Ishii, T. and Adachi, S. (2002) A Case of Mucinous Cystadenocarcinoma of the Appendix Penetrating the Urinary Bladder. Hinyokika Kiyo, 48, 351-354.

[15] Katsuno, G., Kagawa, S., Kokudo, Y., Muraoka, A., Tatemoto, A., Sone, Y., Tsumura, M., Tsuruno, M. and Mizobuchi, K. (2005) Ureteral Metastasis from Appendiceal Cancer: Report of a Case. Surgery Today, 35, 168-171. http://dx.doi.org/10.1007/s00595-004-2876-4

[16] Kamran, A., Hoque, R., El-Tawil, S., Khan, M. and George, M. (2008) Adenocarcinoma of the Appendix Presenting as Bilateral Ureteric Obstruction. World Journal of Surgical Oncology, 6, 23-28. http://dx.doi.org/10.1186/1477-7819-6-23

\section{List of Abbreviations}

CRP: C reactive protein. 